\title{
Reducing the Burden of Ischemia Reperfusion
}

\author{
Editorial to: "Effect of Chronic CPT-1 Inhibition on Myocardial Ischemia-Reperfusion \\ Injury (I/R) in a Model of Diet-Induced Obesity" by G. Maarman et al.
}

\author{
Roger M. Beadle
}

Published online: 14 March 2012

(C) Springer Science+Business Media, LLC 2012

Ischemia reperfusion (I/R) injury relates to the organ damage that results when blood supply is re-instated following an episode is ischemia. This conceptually difficult phenomenon arises from oxidation and inflammation that is triggered by oxidative stress during the period of ischemia after blood flow is restored. Exploration in this field is imperative to better understand the underlying mechanisms of $I / R$, with the hope of translating these findings to the field of cardiology and cardiac surgery. Much effort is directed at minimising and attempting to reverse the deleterious effects of ischemia that result from coronary artery occlusion, cardiac surgical interventions and cardiopulmonary bypass.

Different approaches have been tested to minimize I/R and the most tested and utilised of these is glucose-insulinpotassium (GIK) which has shown benefit outside the realms of poorly controlled diabetes. The utilisation of this cocktail in the setting of critically ill patients [1] and coronary artery by-pass is now accepted clinical practice [2]. Recent evidence has also suggested a role for its use in patients undergoing aortic valve surgery where hypertrophied myocardium is perceivably more sensitive to ischemic insult [3]. The effect of GIK is farther reaching than its effect on glucose and reducing the detrimental consequences of hyperglycaemia. One mechanism of interest is the increase of insulin mediated glucose flux leading to improved oxygen efficiency and cardiac energy provision. Although reperfused hearts are not energy deficient as such, energy substrate preference is an important determinant of the degree $I / R$ injury. This is particularly significant in hearts

R. M. Beadle $(\bowtie)$

University of Aberdeen, School of Medicine and Dentistry,

Polwarth Building,

Foresterhill, Aberdeen AB25 2ZD, UK

e-mail: rogerbeadle@hotmail.com exposed to high levels of fatty acid, which preferentially inhibit glucose oxidation (via inhibition of pyruvate dehydrogenase) and thereby, further fuel the mismatch in glycolysis-glucose oxidation coupling. This prolongs intracellular acidosis (from lactate) during reperfusion, increases the potential for $\mathrm{Na}^{+}$accumulation, $\mathrm{Ca}^{2+}$ overload and left ventricular systolic dysfunction [4]. Obesity is one such condition whereby the heart is exposed to high level of circulating fatty acids making it theoretically more susceptible to $\mathrm{I} / \mathrm{R}$.

Metabolic modulating agents are postulated to act by blocking the metabolism of oxygen demanding fatty acids and up regulating the utilisation of more metabolically efficient glucose, which requires less oxygen to metabolise per unit of ATP produced. Oxfenicine is an irreversible inhibitor of carnitine palmitoyltransferase 1 (CPT-1), a key enzyme in regulating the entry of long chain fatty acids through the mitochondrial outer membrane, by binding them to carnitine. Although it has not been used in human clinical studies, it has gained a place allowing CPT-1 inhibition in animal models testing the effects on fat metabolism.

Some of studies have shown negative outcomes associated with the experimental use of oxfenicine in lean animals $[5,6]$. On the other hand, there is considerable evidence to suggest that oxfenicine is effective in protecting the isolated perfused rat heart against from demand induced ischaemia and in delaying the onset of heart failure in a paced animal model $[7,8]$.

There is some debate as to the utility of blocking fatty acid metabolism especially in the case of a long chain saturated fat diet. In one of the better studies in this area Okere et al. [9] hypothesised that CPT-1 inhibition would be more detrimental to cardiac function in mice fed a diet high in long-chain fatty acids. This is because long-chain fatty acids are dependent on CPT- 1 to enter the mitochondrion 
and blocking this step would lead to an accumulation of triglyceride and lipid by products in the cytosol causing hypertrophy and impaired cardiac function. They found that there was indeed a lipid accumulation in CPT-1 inhibited mice fed saturated fats, but this did not result in cardiac hypertrophy or dysfunction. There was no test as to the effects of $\mathrm{I} / \mathrm{R}$ in this study but it showed that these potential problems with oxfenicine and obesity were not detrimental, at least in the short term. Longer duration animal studies have shown significant accumulation and myocardial hypertrophy with oxfenicine use [10].

With this background, Maarman et al. [11] explored in this issue of Cardiovascular Drugs and Therapy the effect of CPT-1 inhibition in I/R, in the setting of high circulating free fatty acid levels induced by obesity, in a rat model, comparing results to a control group. The obesity was achieved by feeding the animals a high calorie diet with modestly more carbohydrate and fat than standard chow for 16 weeks. Despite this seemingly modest change to diet, the rats achieved a highly significant weight gain with much more retroperitoneal fat. Levels of circulating free fatty acids were not measured.

Rate pressure product (RRP) recovery was used as the index of cardiac mechanical function. Oxfenicine or obesity had no effect on pre-ischaemic function, which is reassuring and consistent with previous work in this area. As expected, and consistent with their hypothesis, oxfenicine was beneficial to the obese animals compared with controls. This improvement was not associated with changes in cardiac mitochondrial respiration. Interestingly, oxfenicine proved detrimental when compared with control, in lean animals with respect to RRP recovery and infarct size. This was associated with suppressed cardiac mitochondrial respiration.

These findings are thought provoking and may relate to a result of the systemic effect of CPT-1 inhibition or other mechanisms of action of oxfenicine, which are more pronounced in the lean animal. The authors go some way to explain this by demonstrating a higher insulin receptor substrate-1 (IRS-1) expression in the oxfenicine treated control group compared with untreated controls and lower in the oxfenicine treated obese group compared to the untreated obese group. This could conceivably leave the myocytes more susceptible to ischemia due to severe suppression of insulin signalling.
This study is another piece in the vast puzzle that is the understanding of the actions of metabolic modulating agents. Given the accumulating evidence that the effects of these agents may be transformed in the setting of obesity and altered diet, careful consideration needs to this fact when planning studies using CPT-1 inhibitors, whatever the setting. The effect of oxfenicine in reducing the burden of $\mathrm{I} / \mathrm{R}$ in this model of obesity fuels hope that these agents may one day be useful clinical agents.

\section{References}

1. Van den Berghe G, Wouters P, Weekers F, et al. Intensive insulin therapy in critically ill patients. N Engl J Med. 2001;345:1359-67.

2. Ingels C, Debaveye Y, Milants I, et al. Strict blood glucose control with insulin during intensive care after cardiac surgery: impact on 4-years survival, dependency on medical care, and quality-of-life. Eur Heart J. 2006;27:2716-24.

3. Howell NJ, Ashrafian H, Drury NE, et al. Glucose-insulinpotassium reduces the incidence of low cardiac output episodes after aortic valve replacement for aortic stenosis in patients with left ventricular hypertrophy: results from the Hypertrophy, Insulin, Glucose, and Electrolytes (HINGE) trial. Circulation. 2011;123:170-7.

4. Liu Q, Docherty JC, Rendell JC, et al. High levels of fatty acids delay the recovery of intracellular $\mathrm{pH}$ and cardiac efficiency in post-ischemic hearts by inhibiting glucose oxidation. J Am Coll Cardiol. 2002;39:718-25.

5. Bachmann E, Weber E. Biochemical mechanisms of oxfenicine cardiotoxicity. Pharmacology. 1988;36:238-48.

6. Jodalen H, Ytrehus K, Moen P, et al. Oxfenicine-induced accumulation of lipid in the rat myocardium. J Mol Cell Cardiol. 1988;20:277-82.

7. Chandler MP, Chavez PN, McElfresh TA, et al. Partial inhibition of fatty acid oxidation increases regional contractile power and efficiency during demand-induced ischemia. Cardiovasc Res. 2003;59:143-51.

8. Lionetti V, Linke A, Chandler MP, et al. Carnitine palmitoyl transferase-I inhibition prevents ventricular remodeling and delays decompensation in pacing-induced heart failure. Cardiovasc Res. 2005;66:454-61.

9. Okere IC, Chandler MP, McElfresh TA, et al. Carnitine palmitoyl transferase-I inhibition is not associated with cardiac hypertrophy in rats fed a high-fat diet. Clin Exp Pharmacol Physiol. 2007;34:113-9.

10. Greaves P, Martin J, Michel MC, et al. Cardiac hypertrophy in the dog and rat induced by oxfenicine, an agent which modifies muscle metabolism. Arch Toxicol Suppl. 1984;7:488-93.

11. Maarman G, Marais E, Lochner A, Du Toit EF. Effect of chronic CPT-1 inhibition on myocardial ischemia-reperfusion injury (I/R) in a model of diet-induced obesity. Cardiovasc Drugs Ther 2012;26:this issue 